\title{
THE EVOLUTION OF AN AVERAGE SOLAR GRANULE
}

\author{
R.C. Al tróck \\ Air Force Geophysics Laboratory \\ Sunspot NM, U.S.A.
}

Abstract

High-resolution photographic spectra of the center of the solar disk have been obtained with the Vacuum Tower Telescope at Sacramento Peak Observatory. Two weak iron lines and the neighboring continuum were recorded with 40 sec time resolutions and better than 1" spatial resolution over a period of $40 \mathrm{~min}$. Intensity and velocity fluctuations were obtained in the two lines and continuum as a function of time and space, and $300 \mathrm{sec}$ oscillations were filtered out. The resulting fluctuations, due solely to granulation, were assembled into an ensemble average of the center of a granule and the center of an intergranular lane, as a function of time. The intensity-fluctuation data have been analyzed through calculation of model line profiles to yield temperature fluctuations in a granule as functions of time and height. We find that the line parameters are distinctly out of phase with continuum brightness, so that, for example, maximum brightness at line center occurs approximately $100 \mathrm{sec}$ prior to maximum continuum brightness. A series of one-dimensional model atmospheres representing the granule at various stages of its lifetime is presented.

Dainis Dravins

Lund Observatory

S-222 24 Lund, Sweden

Abstract

Convective motions are manifest in the solar spectrum as slight spectral line asymmetries and wavelength shifts. These have been studied for $311 \mathrm{Fe} I$ lines. Most lines are blueshifted because the larger contribution of blueshifted photons from 
bright and rising granules statistically dominates over the contribution from dark and sinking intergranular lanes. Fainter lines (formed deeper) show larger blueshifts than strong lines; high-excitation lines usually are more blueshifted (preferentially formed in the hotter granules) and short-wavelength lines are more blueshifted because of increased granulation contrast there. Detailed studies of line bisector behavior as function of line parameters permit the construction of model atmospheres incorporating convective hydrodynamics. The method does not require spatially resolved observations and can be extended to studies of stellar convection.

\section{DIFFERENTIAL LINE SHIFTS IN IATE TYPE STARS}

\section{A. Stawikowski}

N. Copernicus Astronomical Center, Astrophysics Laboratory

Torun, Poland

\section{Abstract}

The differential line shifts method for studying convective type motions has been described in the review of Glebocki and stawikowski at this colloquium. Here the results of differential line shifts for twenty late type stars are presented. Line shifts were determined either from published wavelength measurements ( $\alpha \mathrm{CMi}, \beta \mathrm{Peg}$, $\alpha$ Boo, $\alpha$ Ser, $\alpha$ UMa, $\alpha$ Per, $\alpha$ SCo, $\gamma$ CYg, $\alpha$ Car and HD 19445) or from my own wavelength measurements on $8.1 \mathrm{~A} / \mathrm{mm}$ dispersion spectra (56 Ori, $\eta$ Peg, $\eta$ PsC, 61 Cyg A, $\varepsilon$ Eri, $\delta$ Eri, $\circ$ Tau, $\varepsilon$ Cyg, $\alpha$ Aqr and 1 Ari). Because of low accuracy of the wavelength determinations, mean shifts (VR) were calculated for narrow ranges of lower excitation potentials (LEP). These shifts were plotted against excitation potential and VR-LEP relations were obtained for all analysed stars. Because of the scatter of points in these diagrams linear approximation was assumed and the slopes (denoted $A$ and expressed in $\mathrm{km} / \mathrm{s} / \mathrm{eV}$ ) of the VR-IEP relations were calculated. The stellar parameters and the derived slopes for the whole sample of investigated stars were analysed, and correlations with microturbulence, luminosity and gravity were found. These correlations demonstrate that the slope of VR-IEP relation describes some component of velocity field in late type stars.

The detailed description of the procedure and results will be published in Acta Astronomica. 